\section{Proposta de um observatório epidemiológico do Sistema Único de Saúde}

\author{
Proposal for an epidemiological observatory in \\ the Brazilian Unified National Health System
}

\section{Propuesta de un observatorio epidemiológico del Sistema Único de Salud brasileño}

\section{Resumo}

Após a criação do Sistema Único de Saúde (SUS), o Departamento de Informática do SUS (DATASUS) foi estabelecido em 1991, objetivando a organização de sistemas de informação e bases de dados em saúde. O acesso e visualização dos dados são realizados de forma gratuita e aberta pela Internet, por meio de tabelas e gráficos de dados agregados ou acesso a dados brutos. A presente forma de acesso aos dados não atende completamente a demanda dos gestores e outros usuários por uma ferramenta flexível e de fácil uso, que permita lidar com diferentes aspectos da saúde que sejam relevantes na busca de conhecimento e no processo de decisão. Nós propomos um sistema auxiliar capaz de gerar relatórios mensais sintéticos de maneira automatizada, que sejam facilmente acessados e também de fácil compreensão, com ênfase na visualização de informações valendo-se de gráficos e mapas.

Disseminação de Informação; Algoritmos; Internet; Sistemas de Informação
Raphael de Freitas Saldanha 1 Ronaldo Rocha Bastos 2

Maria Teresa Bustamante-Teixeira 3 Isabel Cristina Gonçalves Leite 3 Estela Márcia Saraiva Campos 3

doi: 10.1590/0102-311X00113216

\section{Correspondência}

R. F. Saldanha

Programa de Pós-graduação em Saúde Coletiva,

Universidade Federal de Juiz de Fora.

Prédio do NATES/UFJF, Campus Universitário $s / n$, Juiz de Fora, MG 36036-900, Brasil.

rfsaldanha@gmail.com

1 Programa de Pós-graduação em Saúde Coletiva, Universidade Federal de Juiz de Fora, Juiz de Fora, Brasil. 2 Instituto de Ciências Exatas, Universidade Federal de Juiz de Fora, Juiz de Fora, Brasil.

3 Faculdade de Medicina, Universidade Federal de Juiz de Fora, Juiz de Fora, Brasil. 


\section{Introdução}

A produção e disponibilização de dados em saúde são fundamentais para a melhoria dos sistemas de saúde, guiando o processo de tomada de decisão e pesquisa ${ }^{1}$. Contudo, a disponibilidade e qualidade de dados sobre saúde em países em desenvolvimento podem ser consideradas, em geral, aquém do necessário 2 , ainda que se reconheçam as diversas dificuldades para a coleta e o processamento de dados nestes países.

No Brasil, com o processo de redemocratização de 1988, a nova Constituição estabeleceu o Sistema Único de Saúde (SUS) e, em 1991, foi criado o Departamento de Informática do SUS (DATASUS) pelo Decreto no 100, de 16 de abril de 1991. Assim, parte da estrutura responsável pela coleta de dados e pelos sistemas de saúde da DATAPREV e outros foram redirecionados para criar a Fundação Nacional de Saúde (FUNASA) e o DATASUS 3.

Com o Decreto no 4.194, de 11 de abril de 2002, a estrutura do Ministério da Saúde foi remodelada, e as competências do DATASUS foram redefinidas. Dentre elas, destacam-se os papéis de manutenção e desenvolvimento de sistemas de informação em saúde, de tecnologias para implementação e disseminação de informações, manutenção do acervo de bases de dados e outras ações 3 (Rede Interagencial de Informações para a Saúde. http://datasus.saude.gov.br/noticias/58-ripsa, acessado em 20/Abr/2015).

Seguindo suas competências, a missão principal do DATASUS está na produção e disseminação de dados e não na análise destes. Uma preocupação recorrente em países em desenvolvimento, além da produção e organização de dados, é a sua própria utilização, levando à necessidade de fortalecimento nesta área 2 .

Uma pesquisa de 2009 4, com usuários do TABNET e TABWIN, aponta a necessidade de maiores investimentos na capacitação para a utilização dos sistemas. Dados de 2014 mostram que 85\% dos estabelecimentos de saúde têm acesso à Internet e 99\% dos médicos acessaram a Internet nos últimos 12 meses 5 . Porém, as estatísticas de acesso ao site do DATASUS apresentam uma média de 210 mil acessos (DATASUS. Estatísticas de acesso ao TABNET: 2014. http://www2.datasus.gov.br/DATASUS/ index.php? area=0209, acessado em 20/Abr/2016). Considerando que existem mais de 200 mil profissionais na área administrativa do SUS (DATASUS. http://www2.datasus.gov.br, acessado em 20/ Abr/2015), o número de acessos ao site pode ser visto como aquém do esperado. Pode-se considerar como um dos fatores determinantes a necessidade de treinamento 5 e de conhecimentos razoáveis de epidemiologia por parte do usuário.

Dessa maneira, seguindo os esforços da coleta de dados, maior atenção deve ser dedicada à comunicação de dados e indicadores. A disseminação de dados para gestores e tomadores de decisão necessita ir além de indicadores simples e valores brutos, apresentando maior ênfase em suas séries históricas e na relação com outros indicadores, estabelecendo relações entre subgrupos e áreas geográficas, usando gráficos e mapas como meios de comunicação 6 .

Esta proposta de criação de um observatório de dados do SUS objetiva contribuir para o atendimento da demanda de gestores, pesquisadores e da população em geral por informações de saúde, propondo uma ferramenta flexível e de fácil uso, não demandando treinamento específico, e que possa lidar com os diferentes aspectos que sejam relevantes na busca de conhecimento e no processo de decisão.

A proposta de observatórios de saúde pública iniciou na França em 1974, com a necessidade de oferta de informações que permitisse a utilização direta para a formulação de políticas públicas, com ênfase na organização e sintetização dos dados já existentes no país. Essa proposta apresentou consideráveis avanços com o desenvolvimento de observatórios no Reino Unido, a partir de 1990, agregando-se conceitos de análise, crítica e intervenção nos objetivos de observatórios de saúde pública 7,8.

Os observatórios de saúde costumam apresentar características em comum, em que destaca-se o fato de que são iniciativas em geral propostas e mantidas por um pequeno grupo, envolvendo acadêmicos e profissionais, preservando autonomia em relação ao sistema de saúde. Apresentam informações sintetizadas, objetivas e atualizadas, obtidas com base em grande volume de dados para um público não necessariamente especializado 7 . 
Nesse sentido, esta proposta de observatório do SUS está nas fases iniciais de construção ${ }^{9}$, iniciando pela coleta, organização e sintetização de dados existentes, capazes de gerar relatórios mensais sintéticos automatizados que sejam facilmente acessados. Ênfase é colocada na visualização de informações, conforme apresentado a seguir.

\section{Metodologia}

\section{Dados}

O Ministério da Saúde, por intermédio do DATASUS, disponibiliza microdados dos sistemas de informação. Esses microdados são apresentados em estrutura de casos (uma ocorrência por linha).

Esses arquivos são copiados mensalmente, mediante rotinas de leitura de dados, e importados para o software R (The R Foundation for Statistical Computing, Viena, Áustria; http://www.r-project. org). Após a leitura dos microdados, as bases são preparadas descartando-se variáveis não utilizadas (como códigos de identificação do registro), convertendo unidades de medida e identificando as variáveis categóricas já existentes, seguindo os arquivos de documentação do DATASUS. Esses passos são executados de maneira automatizada utilizando-se o algoritmo de download e pré-processamento. Essa etapa resulta na criação de bases de dados separadas por categorias de interesse.

Essas categorias de interesse separam os registros das bases de dados seguindo a 10a revisão da Classificação Internacional de Doenças (CID-10) informada nos campos de diagnóstico. Adotou-se como critério as 22 categorias usadas pelo projeto da Carga Global de Doenças 10. Essas categorias refletem um esforço internacional de agrupamento e padronização de causas de óbitos para a criação de indicadores gerais.

Atualmente, apenas os dados do Sistema de Informações Hospitalares do SUS (SIH-SUS) são usados, mas é prevista a utilização de dados de outros sistemas. A integração desses dados necessariamente irá requerer processos específicos de compatibilização e harmonização das bases.

Optou-se por iniciar o projeto com dados do SIH-SUS devido à maior periodicidade de atualização (mensal, com defasagem média de dois meses), o que possibilita um acompanhamento próximo da dinâmica dos indicadores, um dos principais interesses de observatórios.

\section{Processamento}

O processamento dos dados visa a criar relatórios para cada categoria de interesse e níveis de agregação. Um algoritmo principal foi desenvolvido para a emissão de relatórios atendendo quatro níveis de agregação: nacional, unidades federativas, macro e microrregiões de saúde.

A criação de relatórios é feita pelas bibliotecas do ambiente R (https://cran.r-project.org/web/ packages/knitr/index.html, acessado em 09/Mai/2015; https://cran.r-project.org/web/packages/ markdown/index.html, acessado em 22/Abr/2015), sendo esta a etapa de maior tempo de processamento. Sempre que possível, estratégias de programação mais velozes são empregadas (https:// cran.r-project.org/web/packages/doParallel/index.html, acessado em 14/Out/2015).

Todos os algoritmos do projeto são organizados em um repositório de versões de código, permitindo o controle e recuperação de versões, seguindo recomendações preconizadas sobre reprodutibilidade de pesquisas 11 .

\section{Resultados}

A metodologia citada foi implantada gerando um protótipo, cujos resultados são disponibilizados pelo site: http://observasus.org. A arquitetura do site foi criada de modo a apresentar uma interface atrativa e simples, exigindo do usuário um mínimo conhecimento de epidemiologia. O site é confeccionado e mantido pelos autores. Financiamentos junto a entidades de fomento estão sendo buscados. 
Os relatórios são acessados diretamente na primeira página (http://observasus.org). Após escolher o nível de agregação desejado, o usuário seleciona uma categoria para a visualização do relatório, requerendo no máximo quatro clicks para exibir um relatório. Exemplo de parte do relatório é apresentado na Figura 1.

O site contém outras seções: (1) blog, utilizado para informar aos usuários sobre atualizações, melhorias e divulgação do projeto; (2) seção sobre o projeto, com informações gerais, equipe, tecnologia e metodologias empregadas; (3) downloads, permitindo acesso às bases de dados; e (4) sistema de rastreio de tickets, em que os usuários podem enviar sugestões e relatar erros.

Considerando que a geração dos relatórios se dá de forma automática, duas ações são propostas para o controle da qualidade dos mesmos: (1) os usuários serão incentivados a comunicar erros e sugestões por meio do sistema de tickets; e (2) uma amostra dos relatórios emitidos será analisada manualmente pela equipe do projeto.

\section{Conclusões}

O presente projeto permite prover relatórios informativos para diversas categorias de interesse e níveis de agregação, contribuindo para diminuir a distância entre a disponibilidade de dados e a geração de informações, não agindo como um substituto do DATASUS, mas como uma janela de visualização dos dados informativa e independente.

Mais avanços estão programados, como a utilização de dados de outros sistemas de informação, buscando-se apresentar relatórios mais abrangentes e que reflitam o estado geral de saúde da população. O consequente aumento de informações produzidas irá requerer melhorias na interface dos relatórios de modo a não exigir do usuário conhecimentos aprofundados. Dessa forma, ao disponibilizar informações sobre saúde valendo-se de relatórios atualizados e de fácil compreensão, este projeto se apresenta como uma fonte confiável de informações para gestores, profissionais e para a população.

Figura 1

Exemplo de relatório.

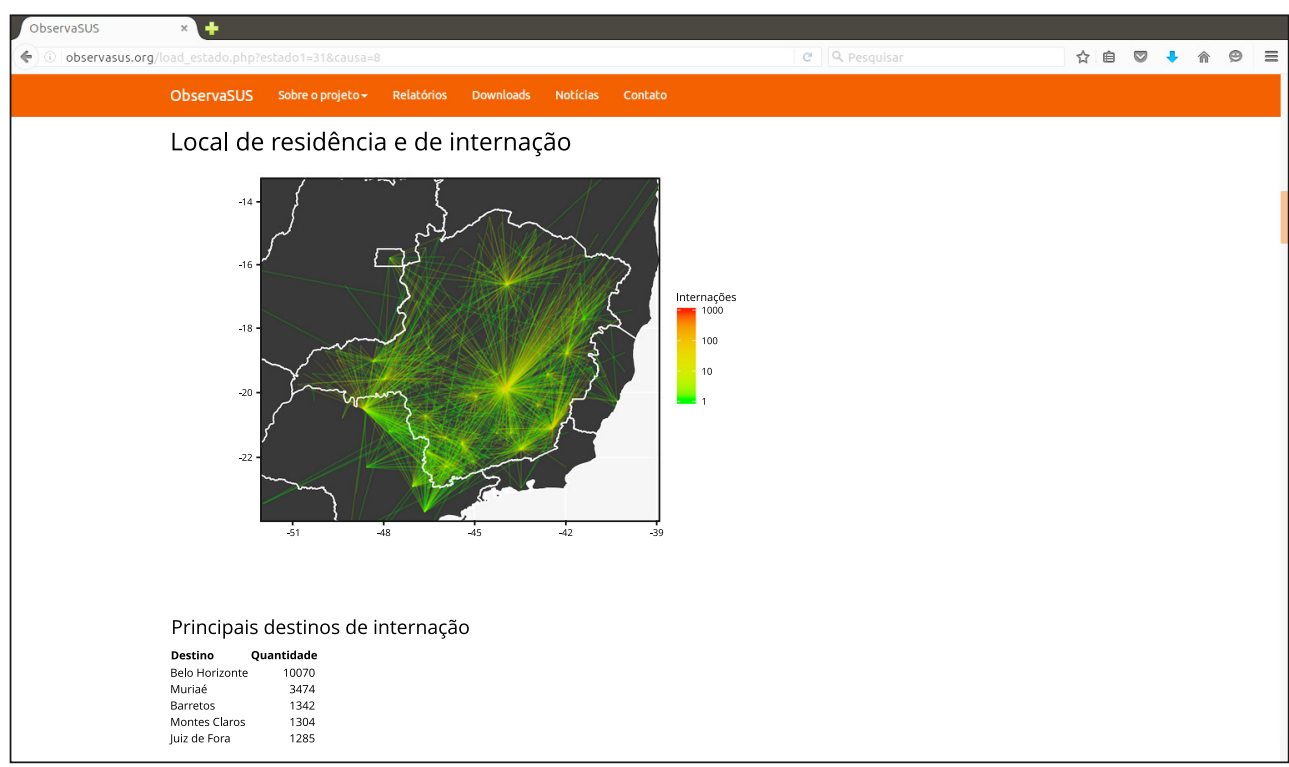




\section{Colaboradores}

R. F. Saldanha, R. R. Bastos, M. T. Bustamante-Teixeira, I. C. G. Leite e E. M. S. Campos contribuíram na concepção do projeto, redação e revisão crítica do artigo.

\section{Agradecimentos}

Este projeto recebe o suporte da Universidade Federal de Juiz de Fora por meio do Programa de Pós-graduação em Saúde Coletiva da Faculdade de Medicina, do Laboratório de Informações Georreferenciadas (LINGE) do Departamento de Estatística e do Núcleo de Assessoria, Treinamento e Estudos em Saúde (NATES).

\section{Referências}

1. Handley K, Boerma T, Victora C, Evans TG. An inflection point for country health data. Lancet Glob Health 2015; 3:e437-8.

2. Boerma T, Stansfield SK. Health statistics now: are we making the right investments? Lancet 2007; 369:779-86.

3. Ministério da Saúde. DATASUS: trajetória 19912002. Brasília: Ministério da Saúde; 2002.

4. Silva NP. A utilização dos programas TABWIN e TABNET como ferramentas de apoio à disseminação das informações em saúde [Dissertação de Mestrado]. Rio de Janeiro: Escola Nacional de Saúde Pública Sergio Arouca, Fundação Oswaldo Cruz; 2009.

5. Comitê Gestor da Internet no Brasil. TIC Saúde 2014. São Paulo: Comitê Gestor da Internet no Brasil; 2015.

6. AbouZahr C, Adjei S, Kanchanachitra C. From data to policy: good practices and cautionary tales. Lancet 2007; 369:1039-46.
7. Hemmings J, Wilkinson J. What is a public health observatory? J Epidemiol Community Health 2003; 57:324-6.

8. Wilkinson J. Public health observatories in England: recent transformations and continuing the legacy. Cad Saúde Pública 2015; 31 Suppl 1:S26976.

9. Castillo-Salgado C. Developing an academiabased public health observatory. Cad Saúde Pública 2015; 31 Suppl 1:S286-93.

10. Department of Health Statistics and Information Systems, World Health Organization. WHO methods and data sources for global burden of disease estimates 2000-2011. Geneva: World Health Organization; 2013. (Global Health Estimates Technical Paper WHO/HIS/HSI/GHE/2013.4).

11. Kass RE, Caffo BS, Davidian M, Meng X-L, Yu B, Reid N. Ten simple rules for effective statistical practice. PLoS Comput Biol 2016; 12:e1004961. 


\section{Abstract}

Following the creation of the Brazilian Unified National Health System (SUS), the Brazilian Health Informatics Department (DATASUS) was established in 1991, aimed at organizing information systems and databases in health. Online data access and viewing is free and open, using tables and graphs of aggregate data and access to raw data. However, the current form of data access does not fully meet the demands by health system administrators and other users for a flexible, user-friendly tool that allows dealing with various relevant health issues in the knowledge search and decision-making. We propose an ancillary system capable of generating monthly summary reports that are easy to access and understand, with an emphasis on viewing information through graphs and maps.

Information Dissemination; Algorithms; Internet; Information Systems

\section{Resumen}

Tras la creación del Sistema Único de Salud (SUS) en Brasil, se estableció en 1991 el Departamento de Informática del SUS (DATASUS), con el objetivo de organizar sistemas de información y bases de datos en salud. El acceso y visualización de los datos se realizan de forma gratuita y abierta, vía Internet, mediante tablas y gráficos de datos agregados o de acceso a datos brutos. La presente forma de acceso a los datos no atiende completamente la demanda de los gestores y otros usuarios de una herramienta flexible y de fácil uso, que permita enfrentarse a diferentes aspectos de la salud que sean relevantes en la búsqueda de conocimiento y en el proceso de decisión. Nosotros proponemos un sistema auxiliar capaz de generar informes mensuales sintéticos, de manera automatizada, que sean de fácil acceso y también de fácil comprensión, con énfasis en la visualización de información, valiéndose de gráficos y mapas.

Diseminación de Información; Algoritmos;

Internet; Sistemas de Información
Recebido em 29/Jun/2016

Versão final reapresentada em 27/Set/2016 Aprovado em 11/Out/2016 